\title{
Black Hole Atom as a Dark Matter Particle Candidate
}

\author{
V. I. Dokuchaev and Yu. N. Eroshenko \\ Institute for Nuclear Research of the Russian Academy of Sciences, 60th October Anniversary Prospect 7a, Moscow 117312, Russia \\ Correspondence should be addressed to Yu. N. Eroshenko; eroshenko@ms2.inr.ac.ru
}

Received 29 November 2013; Revised 28 January 2014; Accepted 28 January 2014; Published 5 March 2014

Academic Editor: Maxim Khlopov

Copyright (C) 2014 V. I. Dokuchaev and Yu. N. Eroshenko. This is an open access article distributed under the Creative Commons Attribution License, which permits unrestricted use, distribution, and reproduction in any medium, provided the original work is properly cited. The publication of this article was funded by SCOAP ${ }^{3}$.

\begin{abstract}
We propose the new dark matter particle candidate-the "black hole atom," which is an atom with the charged black hole as an atomic nucleus and electrons in the bound internal quantum states. As a simplified model we consider the the central ReissnerNordström black hole with the electric charge neutralized by the internal electrons in bound quantum states. For the external observers these objects would look like the electrically neutral Schwarzschild black holes. We suppose the prolific production of black hole atoms under specific conditions in the early universe.
\end{abstract}

\section{Introduction}

The idea of "black hole atoms" goes back a long way in several variations. Markov et al. proposed and studied in detail the model of maximons (or friedmons) [1-3]. These objects are the particle-like gravitating systems (semiclosed worlds) with mass close to the Planck mass $M_{\mathrm{Pl}}=\sqrt{\hbar c / G} \approx 10^{-5} \mathrm{~g}$. They may have in principle a large gravitational mass defect. Maximons are interesting for cosmological applications, in particular, because they have the particle-like properties and may be the enigmatic dark matter particles. The idea of micro black hole carrying the electric charge and having the orbiting electrons or protons at the outer (outside the horizon) orbits was discussed by Hawking in [4]. He firstly proposed that the charged black holes may play a role similar to the atomic nuclei. Later the idea of black hole atoms was investigated in [5-7]. The possible origin of such Planck mass black hole is the final stable state of the evaporated primordial black holes $(\mathrm{PBH})$; see, for example, $[2,3,8]$. The remnants of the evaporated black holes can be stable and also can serve as the dark matter candidates [9-15].

In this paper we discuss the black hole atoms, which are the atoms with the charged black hole as atomic nuclei and with electrons in the bound internal quantum states. The quantum bound states of electrons may exist in principle not only outside the event horizon but also inside the Cauchy horizon of the charged black hole. So, the main new idea is that there can be configurations in which the orbiting electrons are inside the black hole Cauchy horizon. We propose these black hole atoms as the possible origin of dark matter particles.

The quantum levels in the gravitational field of black holes outside the event horizon were studied in [16-27]. The resulting black hole atoms can be the dark matter particles in the case of uncompensated charge (electrons at outer levels) as it was proposed in [26]. The similar idea but for the zero total charge $q=-Q$ and for the electrons inside a black hole was proposed in [28]. In the latter case, the total charge of all the electrons at the inner orbits is equal to the charge of the black hole, which appears at Reissner-Nordström metric. In the case of compensated charge these systems look for the external observer as having the Schwarzschild metric. Neutral systems interact weakly with other particles, it makes them the good candidates for the dark matter particles.

The stationary quantum levels of fermions in the gravitational field of the charged black holes have been found in the work [28] by solving the corresponding Dirac equation. The Dirac equation in the Riemann geometry was first derived in the paper [29]. The using of only the covariant generalization is not enough for derivation of the corresponding Dirac equation. It is needed the determination of the parallel spinor transport. As it was shown in [28], a self-consistent steadystate solution with a finite normalization integral can exist only in the case of extreme black hole, whose charge in the appropriate units is equal to its mass $M=|Q|$. 


\section{Electrons inside the Black Holes}

Let us briefly describe the method of quantum level calculations (for more details see [28]). The Dirac equation in the general metric has the following form [30]:

$$
\left(i \gamma^{\mu} D_{\mu}-m\right) \psi=0
$$

where $\gamma^{\mu}=e_{(a)}{ }^{\mu} \gamma^{(a)}, \gamma^{(a)}$ are the standard Dirac matrices, and $e_{(a)}{ }^{\mu}$ are tetrads. The elongated derivative has the form $D_{\mu}=\partial_{\mu}+i q A_{\mu}+\Gamma_{\mu}$, where $\Gamma_{\mu}=(1 / 4) \gamma^{(a)} \gamma^{(b)} e_{(a)}{ }^{\mu} e_{(b) v ; \mu}$, and $A_{\mu}$ is the electromagnetic 4-potential. We consider a charged Reissner-Nordström black hole with the metric $d s^{2}=f d t^{2}-$ $f^{-1} d r^{2}-r^{2}\left(d \theta^{2}+\sin ^{2} \theta d \phi^{2}\right)$, where $f=1-2 M / r+Q^{2} / r^{2}$, $M$ is the black hole mass, and $Q$ is its charge. Following the method of [30] we separate the variables by the following way:

$$
\psi=e^{-i E t} Z(\theta, \phi) f^{-1 / 4} r^{-1}(\sin \theta)^{-1 / 2}\left[\begin{array}{c}
g(r) I_{2} \\
i h(r) I_{2}
\end{array}\right],
$$

where $I_{2}=(1,1)^{T}$, and $Z(\theta, \phi)$ is the angular part of the wave function. After substitution in (1), we obtain the next system of equations:

$$
\begin{aligned}
& \frac{d g}{d r}-\frac{g k}{r f^{1 / 2}}+\frac{h}{f^{1 / 2}}\left[\frac{1}{f^{1 / 2}}\left(E-\frac{q Q}{r}\right)+m\right]=0, \\
& \frac{d h}{d r}+\frac{h k}{r f^{1 / 2}}-\frac{g}{f^{1 / 2}}\left[\frac{1}{f^{1 / 2}}\left(E-\frac{q Q}{r}\right)-m\right]=0,
\end{aligned}
$$

where $k=0, \pm 1, \pm 2, \ldots$. The condition for the physically acceptable solution is the finiteness of the normalization integral

$$
2 \int_{0}^{r_{-}}\left(|g|^{2}+|h|^{2}\right) f^{-1}(r) d r=1,
$$

which was derived from the zero component of the fermion current $j^{\mu}=\bar{\psi} \gamma^{\mu} \psi$. This integral is finite only in the case of extremal black hole $M=|Q|$. Such a black hole has equal horizons radii $r_{-}=r_{+}=M$. Consider the internal solution for extreme black hole near the Cauchy horizon $r \rightarrow r_{-}$. Let us denote $\mu=m M / M_{\mathrm{Pl}}^{2}$ and $\nu=q Q /(\hbar c)$. As can be shown [28], (3) in this case have the regular solution

$$
g=C\left(\frac{M}{r}-1\right)^{\varkappa}, \quad h=C\left(\frac{M}{r}-1\right)^{\varkappa} \frac{k+\varkappa}{\mu-\nu},
$$

where $C=$ const, $\varkappa=\sqrt{k^{2}+\mu^{2}-v^{2}}$, and (4) is finite in the case $k^{2}+\mu^{2}-v^{2}>1 / 4$. This solution corresponds to the energy level

$$
E=\frac{q Q}{M}
$$

Electron has the same energy of (6) for the regular external solution outside the event horizon. Equation (3) can be solved numerically far away from the horizons [28]. In the case of the nonextreme black hole, $M \neq|Q|$, the integral (4) diverges at the horizons [28].
We suppose that quantum levels in the black hole interiors are stable. These means, additionally, the supposition of the internal universes inside the charged black holes, like in the idealized eternal black holes. The other possibility is the dynamical formation of the internal universes in the gravitational collapse [31]. For the quantum formation of black holes at the particle accelerators the specific extra dimensions are needed [13]. The classical bound stable orbits inside black holes are considered, for example, in [32-34].

The basic condition of existence of an atom as a quantum system in the quasiclassical approximation in flat space is the geometrical condition for Compton wavelength $\lambda \leq a$, where $a$ is the characteristic scale of the potential hole. Strong spacetame curvature, especially near the horizons, changes this criterion drastically. A physical reason for the geometrical criterion breakdown is the infinite stretching of the physical distance $d l^{2}=\left(-g_{\alpha \beta}+g_{0 \alpha} g_{0 \beta} / g_{00}\right) d x^{\alpha} d x^{\beta}$ at the Cauchy horizon $r_{-}$. Really, the physical distance from the centre $r=0$ of the extremal Reissner-Nordström black hole $\left(r_{-}=r_{+}=\right.$ $M$ ) to some $r$ is

$$
l=\int_{0}^{r} f^{-1 / 2} d r=r+M \ln \left(1-\frac{r}{M}\right)
$$

It diverges for $r \rightarrow M$. Therefore, there is enough space under the Cauchy horizon for particles with any wavelength. As seen from the exact solution of the Dirac equation, the wave function of the electron just localised under the horizon, and it occurs far from quasiclassical regime. A question about capture of particle with large $\lambda \gg r_{g}$ wavelength by a black hole can arise. But we do not consider here the capture of the electron inside the black hole. Instead, we propose the formation of black hole with the electron already inside due to single quantum jump.

\section{Black Hole Atoms Formation}

PBHs can be formed at the cosmological stage of radiation dominance from the adiabatic density perturbations $[4,35$, $36]$ and in the early dust-like stages [37, 38]. Although the primordial black hole is formed by the classical gravitational collapse, its final stage of evaporation is mainly the quantum process. Sakharov discussed the possibility of the superheavy particles emission by the black hole at the last stage of its evaporation [39]. The emitted particles could have even the masses up to the Planck mass although the probability of such emission is not clear. One can imagine the last stage of a black hole evaporation not as a gradual radiation but as the quantum jump into a new state. We assume that black hole atoms can result from such quantum jumps. The discussion about the final stage of evaporation can be found in $[40,41]$. In the case of the jump the charged black holes with electrons on internal quantum orbits could be born effectively just after the evaporation of the $\mathrm{PBH}$ population.

Note that the Hawking temperature for the extremal black hole is equal to zero and, so, the extremal black holes do not experience the quantum evaporation (see $[14,15,40,41]$ ), and 
they are stable dark matter candidates in this sense. Really the Hawking temperature of the charged black hole

$$
T_{H} \propto \sqrt{M^{2}-Q^{2}}
$$

equals zero if $Q=M$. Nevertheless, the stability of the extremal black hole atoms with respect to quantum decay remains questionable.

Let us consider in more details the formation mechanism of PBH from the adiabatic density perturbations. Such perturbations can arise at inflationary stage, and the necessary condition for the effective PBH formation is the excess of perturbations' power at some small scale because the simplest near flat spectrum cannot produce the sufficient amount of PBHs. But in the case of some peak in the spectrum, the PBHs can form just in the required cosmological abundance. Now we consider the modification of the required peak's height by taking into account the mass loss of the BPHs during their evaporation from the initial mass $M_{\mathrm{PBH}}$ at the formation moment $t_{f} \simeq G M_{\mathrm{PBH}} / c^{2}$ till the Plank mass remnants after evaporation. With this mass loss the contemporary cosmological parameter of the PBHs can be expressed as [8]

$$
\Omega_{m} \simeq \beta \frac{a\left(t_{\mathrm{eq}}\right)}{a\left(t_{f}\right)} \frac{M_{\mathrm{Pl}}}{M_{\mathrm{PBH}}},
$$

where $\Delta_{h}$ is the r.m.s. perturbation at the mass scale $M_{\mathrm{PBH}}$, $\delta_{c}=1 / 3$ according to the analytical estimation of [36] or $\delta_{c} \simeq 0.7$ as was obtained in the models of critical collapse, $a(t)$ is the scale factor, $t_{\mathrm{eq}}$ is the matter radiation equality moment, and

$$
\beta=\int_{\delta_{c}}^{1} \frac{d \delta}{\sqrt{2 \pi} \Delta_{h}} e^{-\delta^{2} /\left(2 \Delta_{h}^{2}\right)} \simeq \frac{\Delta_{h}}{\delta_{c} \sqrt{2 \pi}} e^{-\delta_{c}^{2} /\left(2 \Delta_{h}^{2}\right)}
$$

By solving iteratively the system of (9) and (10) with $\Omega_{m}=$ 0.3 we find the dependence of $\Delta_{h}$ on $M_{\mathrm{PBH}}$, which is shown at Figure 1. For $M_{\mathrm{PBH}} \geq 10^{13} \mathrm{~g}$ there are no solutions of (9) and (10), and the scenario is limited only by smaller $M_{\mathrm{PBH}}$. Therefore, to produce the cosmological abundance of $\mathrm{PBHs}$ Plank mass remnants $\Omega_{m} \approx 0.3$ as is required for dark matter, one needs the density perturbations spectrum with the r.m.s. values shown at Figure 1.

\section{Interactions of the Black Hole Atoms}

The interaction of the neutral black hole atoms with ordinary matter via the gravitational dynamical friction effect is extremely weak, as it was first shown in [4]. This is due to the extremely small cross-section $\sim \pi r_{g}^{2}(c / v)^{2} \sim 3 \times$ $10^{-66}(c / v)^{2} \mathrm{sm}^{2}$, where $v$ is the relative velocity, $(c / v)^{2} \sim 10^{6}$ in the galactic halo. One can compare it with the neutrinonucleons interaction cross-sections $\sim 10^{-43}-10^{-34} \mathrm{sm}^{2}$.

The black hole can be born charged even in the classical collapse. But the neutralization by the accretion could reduce the initial charge till the value $Z \simeq 30$ [4]. The remaining charge can interact with the ordinary matter in the universe like heavy atomic nuclei [4]. So, the charged black hole

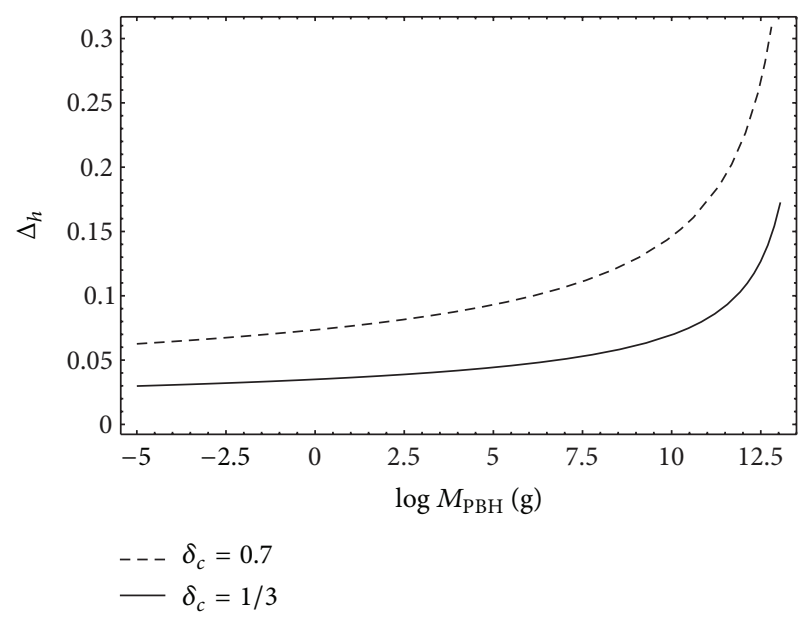

FIgURE 1: The dependence of the r.m.s. perturbation $\Delta_{h}$ at the mass scale $M_{\mathrm{PBH}}$ required for the explanations of dark matter as $\mathrm{PBHs}$ remnants in dependence on the initial PBH's mass $M_{\mathrm{PBH}}$ for value of the collapse threshold $\delta_{c}=1 / 3$ (solid curve) and $\delta_{c} \simeq 0.7$ (dashed curve).

atoms experience strong collisions and dissipations. This creates some difficulties for these systems as dark matter particles. Although, the solution of this problem may be in the formation of molecular-like systems with ordinary charged particles as in the composite-dark-matter scenario [42]. Possible detection method of charged black hole atoms was also proposed in the seminal paper [4]. These systems can draw straight tracks in the chambers almost without the deflection by the magnetic field due to their large masses.

Although in the case of a nonextreme black hole the normalization integral diverges, the irregular quasistationary solutions can exist $[43,44]$. In addition, the regular internal (under the Cauchy horizon) and external solutions are possible [28]. Regular internal solutions have only single energy levels inside and outside the black hole. The internal level has the energy $E=q Q / r_{-}$, and the energy for the outer one is $E=q \mathrm{Q} / r_{+}$. If electron jumps or tunnels from the external to the internal level, the energy

$$
\Delta E=q Q\left(\frac{1}{r_{-}}-\frac{1}{r_{+}}\right)=\frac{2 M c^{2} q}{Q} \sqrt{1-\frac{Q^{2}}{G M^{2}}}
$$

can be radiated in the form of photons. This energy could reach the very high value up to the energy corresponding to the ultrahigh energy cosmic rays. Note that the radiation from the bound system of two friedmons was considered in [2]. The released energy is supplied by the gravitational energy of the black hole and by the energy of the electrostatic interaction of electrons with a charged black hole. The gravitational factor is due to the fact that gravity is responsible for the localization of electrons near horizons at the quasistationary regular quantum orbits. In the case of the extreme black hole one has $r_{+}=r_{-}$. In this case the energy release during the electron transition is absent. For this reason the extreme black holes with $q=-Q$ are the very 
"quiet," dark, and noninteracting objects. These properties are just one needs for the dark matter candidates.

\section{Conclusions}

In this paper we discuss the new kind of "black hole atom" system: the Reissner-Nordström black holes with the electrons at quantum levels under the Cauchy horizon. If the electric charge of the black hole is neutralized by the internal electrons in bound quantum states, these objects would look like the electrically neutral Schwarzschild black holes for the external observers. Due to extremely small interaction crosssection, these neutral systems are almost noninteracting with baryons and behave as collisionless and dissipationless gas. This property makes them the good dark matter candidates.

The black hole atoms under consideration could form at the final stages of PBHs evaporation at early universe. The $\mathrm{PBH}$ itself may form in different scenarios: from adiabatic perturbations, during cosmological phase transitions, or at the early dust-like stages [37]. The extremal black holes do not evaporate in the Hawking process, and they are stable in this sense. But the existence of the internal and external quantum levels gives the possibility of the quantum transitions between the levels with radiation of the photons, and this effect makes the "black hole atoms" observable in principle.

\section{Conflict of Interests}

The authors declare that there is no conflict of interests regarding the publication of this paper.

\section{Acknowledgments}

This study was supported by the Grants RFBR 13-02-00257-a and OFN-17.

\section{References}

[1] M. A. Markov, "Elementary particles of maximally large masses (Quarks and Maximons)," Soviet Physics (Journal of Experimental and Theoretical Physics), vol. 24, p. 584, 1967.

[2] V. I. Man'ko and M. A. Markov, "Properties of fridmons and the early stage of evolution of the universe," Theoretical and Mathematical Physics, vol. 17, no. 2, pp. 1060-1063, 1973.

[3] M. A. Markov, "The maximon and minimon in light of a possible formulation of the concept of an 'elementary particle"' JETP Letters, vol. 45, pp. 141-144, 1987.

[4] S. Hawking, "Gravitationally collapsed objects of very low mass," Monthly Notices of the Royal Astronomical Society, vol. 15, p. 75, 1971.

[5] V. V. Flambaum and J. C. Berengut, "Atom made from charged elementary black hole," Physical Review D, vol. 63, no. 8, Article ID 084010, 2001.

[6] E. G. Floratos, G. K. Leontaris, and N. D. Vlachos, "Gravitational atom in compactified extra dimensions," Physics Letters $B$, vol. 694, no. 4-5, pp. 410-416, 2011.

[7] M. L. Fil'Chenkov and P. Yu. Laptev, "Graviatom dipole radiation," Gravitation and Cosmology, vol. 12, p. 65, 2006.
[8] B. J. Carr, J. H. Gilbert, and J. E. Lidsey, "Black hole relics and inflation: limits on blue perturbation spectra," Physical Review $D$, vol. 50, no. 8, pp. 4853-4867, 1994.

[9] J. H. Macgibbon, "Can Planck-mass relics of evaporating black holes close the Universe?” Nature D, vol. 329, no. 6137, pp. 308309, 1987.

[10] A. D. Dolgov, P. D. Naselsky, and I. D. Novikov, "Gravitational waves, baryogenesis, and dark matter from primordial black holes," http://arxiv.org/abs/astro-ph/0009407 .

[11] R. J. Adler, P. Chen, and D. I. Santiago, "The generalized uncertainty principle and black hole remnants," General Relativity and Gravitation, vol. 33, no. 12, pp. 2101-2108, 2001.

[12] P. Chen and R. J. Adler, "Black hole remnants and dark matter," Nuclear Physics B, vol. 124, pp. 103-106, 2003.

[13] B. J. Carr, "Primordial black holes as a probe of cosmology and high energy physics," Lecture Notes in Physics, vol. 631, pp. 301321, 2003.

[14] I. Dymnikova and E. Galaktionov, "Vacuum dark fluid," Physics Letters B, vol. 645, no. 4, pp. 358-364, 2007.

[15] I. Dymnikova and M. Korpusik, "Regular black hole remnants in de Sitter space," Physics Letters B, vol. 685, no. 1, pp. 12-18, 2010.

[16] N. Deruelle and R. Ruffini, "Quantum and classical relativistic energy states in stationary geometries," Physics Letters B, vol. 52, no. 4, pp. 437-441, 1974.

[17] I. M. Ternov, V. R. Khalilov, G. A. Chizhov, and A. B. Gaina, "Finite motion of massive particles in the Kerr and Schwarzschild fields," Soviet Physics Journal, vol. 21, pp. 12001204, 1978.

[18] L. A. Kofman, "Bound states in quantum evaporation of black holes," Physics Letters A, vol. 87, no. 6, pp. 281-284, 1982.

[19] M. Soffel, B. Muller, and W. Greiner, "Particles in a stationary spherically symmetric gravitational field," Journal of Physics A, vol. 10, no. 4, pp. 551-560, 1977.

[20] I. M. Ternov, A. B. Gaina, and G. A. Chizhov, "Finite motion of electrons in the field of microscopic black holes," Soviet Physics Journal, vol. 23, no. 8, pp. 695-700, 1980.

[21] D. V. Gal'tsov, G. V. Pomerantseva, and G. A. Chizhov, "Filling of quasibound states by electrons in a schwarzchild field," Soviet Physics Journal, vol. 26, no. 8, pp. 743-745, 1983.

[22] I. M. Ternov and A. B. Gaina, "Energy spectrum of the Dirac equation in the Schwarzschild and Kerr fields," Izvestiya Vysshikh Uchebnykh Zavedenil. Fizika, vol. 31, no. 2, pp. 86-92, 1988.

[23] A. B. Gaina and O. B. Zaslavskiř, "On quasilevels in the gravitational field of a black hole," Classical and Quantum Gravity, vol. 9, no. 3, pp. 667-676, 1992.

[24] A. Lasenby, C. Doran, J. Pritchard, A. Caceres, and S. Dolan, "Bound states and decay times of fermions in a Schwarzschild black hole background," Physical Review D, vol. 72, no. 10, Article ID 105014, 2005.

[25] M. V. Gorbatenko and V. P. Neznamov, "Stationary bound states of dirac particles in collapsar fields," http://arxiv.org/abs/1205.4348 .

[26] M. A. Vronsky, M. V. Gorbatenko, N. S. Kolesnikov, V. P. Neznamov, E. Yu. Popov, and I. I. Safronov, "Stationary bound states of dirac particles in the schwarzschild gravitational field," http://arxiv.org/abs/1301.7595.

[27] V. Dzhunushaliev, "Canonical conjugated Dirac equation in a curved space," http://arxiv.org/abs/1202.5100. 
[28] V. I. Dokuchaev and Yu. N. Eroshenko, "Stationary solutions of the dirac equation in the gravitational field of a charged black hole," Journal of Experimental and Theoretical Physics, vol. 117, pp. 72-77, 2013.

[29] V. Fock, "Geometrisierung der diracschen theorie des elektrons," Zeitschrift fur Physik, vol. 57, pp. 261-277, 1929.

[30] D. R. Brill and J. A. Wheeler, "Interaction of neutrinos and gravitational fields," Reviews of Modern Physics, vol. 29, pp. 465479, 1957.

[31] V. P. Frolov, M. A. Markov, and V. F. Mukhanov, "Black holes as possible sources of closed and semiclosed worlds," Physical Review D, vol. 41, no. 2, pp. 383-394, 1990.

[32] J. Bičák, Z. Stuchlík, and V. Balek, "The motion of charged particles in the field of rotating charged black holes and naked singularities," Bulletin of the Astronomical Institutes of Czechoslovakia, vol. 40, pp. 65-92, 1989.

[33] S. Grunau and V. Kagramanova, "Geodesics of electrically and magnetically charged test particles in the Reissner-Nordström space-time: Analytical solutions," Physical Review D, vol. 83, no. 4, Article ID 044009, 2011.

[34] V. I. Dokuchaev, "Is there life inside black holes?" Classical and Quantum Gravity, vol. 28, no. 23, Article ID 235015, 2011.

[35] Ya. B. Zel'dovich and I. D. Novikov, "The hypothesis of cores retarded during expansion and the hot cosmological model," Soviet Astronomy, vol. 10, p. 602, 1967.

[36] B. J. Carr, "The primordial black hole mass spectrum," The Astrophysical Journal, vol. 201, pp. 1-19, 1975.

[37] A. G. Polnarev and M. Yu. Khlopov, "Cosmology, primordial black holes, and supermassive particles," Soviet Physics Uspekhi, vol. 28, p. 213, 1985.

[38] N. A. Zabotin, P. D. Naselskii, and A. G. Polnarev, "Highamplitude peaks of density disturbances and the formation of primordial black-holes in the dust like universe," Soviet Astronomy, vol. 31, p. 353, 1987.

[39] A. D. Sakharov, "Evaporation of black miniholes, highenergy physics," Soviet Journal of Experimental and Theoretical Physics Letters, vol. 44, p. 379, 1986.

[40] I. G. Dymnikova, "De Sitter-Schwarzschild black hole: its particlelike core and thermodynamical properties," International Journal of Modern Physics D, vol. 5, no. 5, pp. 529-540, 1996.

[41] Y. S. Myung, Y.-W. Kim, and Y.-J. Park, "Quantum cooling evaporation process in regular black holes," Physics Letters B, vol. 656 , no. $4-5$, pp. 221-225, 2007.

[42] N. Mankoc Borstnik, H. B. Nielsen, C. D. Froggatt, and D. Lukman, Eds., "Proceedings of the 14th International Workshop, 'What Comes Beyond the Standard Model,' vol. 12, pp. 94-102, Bled, Slovenia, 2011.

[43] M. V. Gorbatenko and V. P. Neznamov, "Stationary bound states of spin-half particles in the Reissner-Nordstroem gravitational field," http://arxiv.org/abs/1302.2557.

[44] M. V. Gorbatenko and V. P. Neznamov, "Stationary bound states of spin-half particles in the Kerr and Kerr-Newman gravitational fields," http://arxiv.org/abs/1303.1127. 

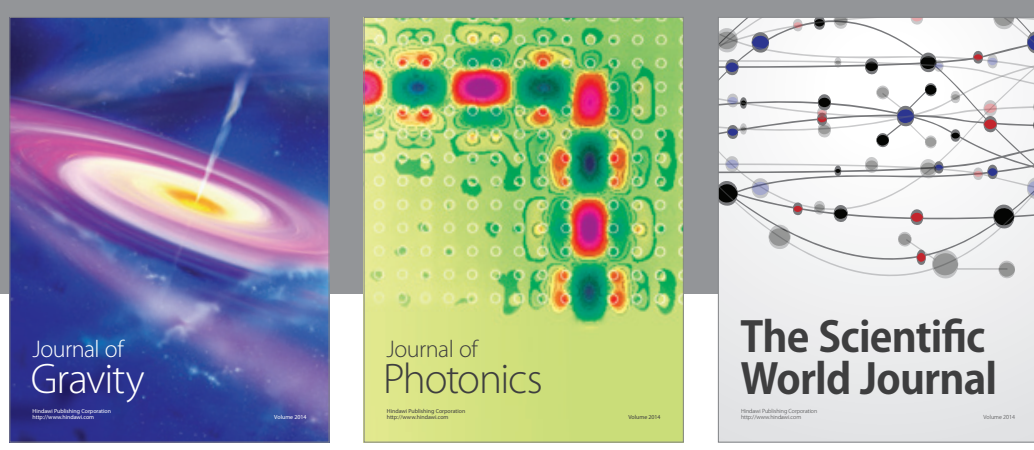

The Scientific World Journal
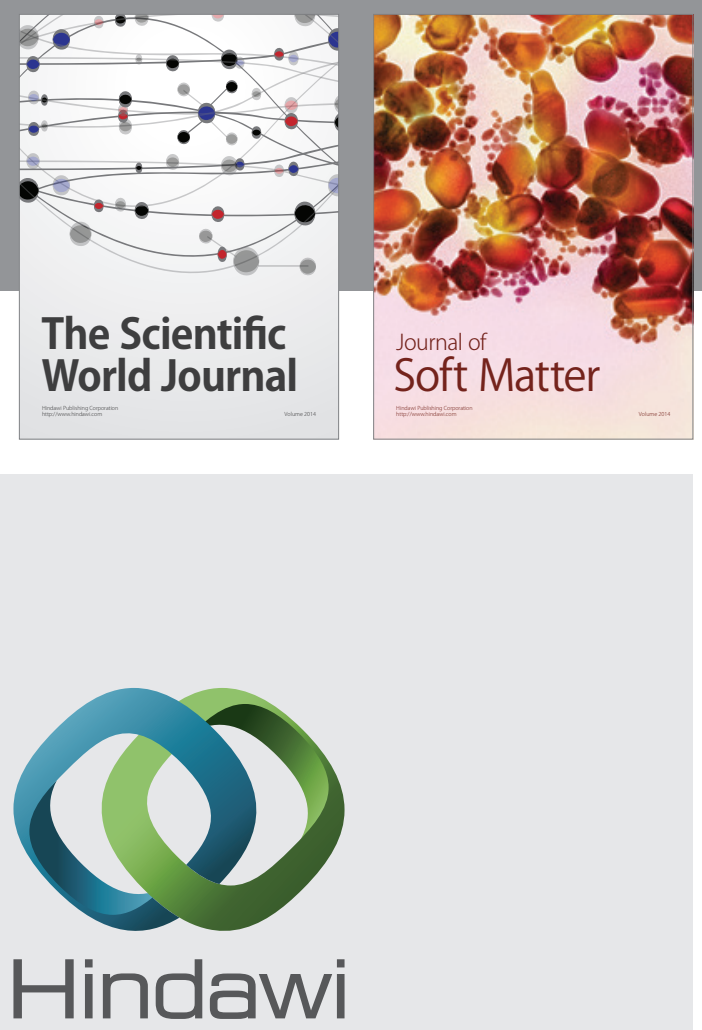

Submit your manuscripts at

http://www.hindawi.com

nternational Journal of

Statistical Mechanics
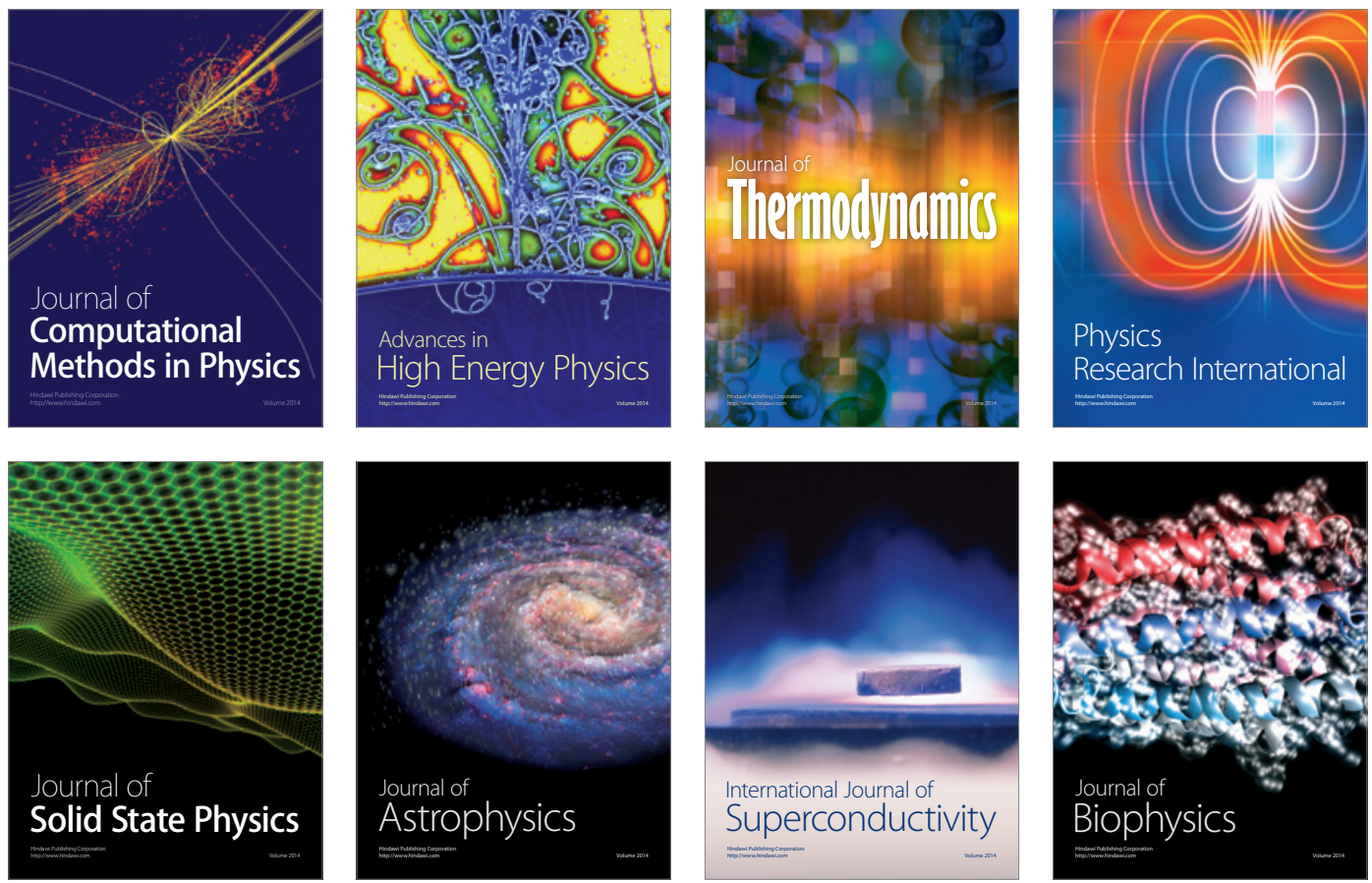
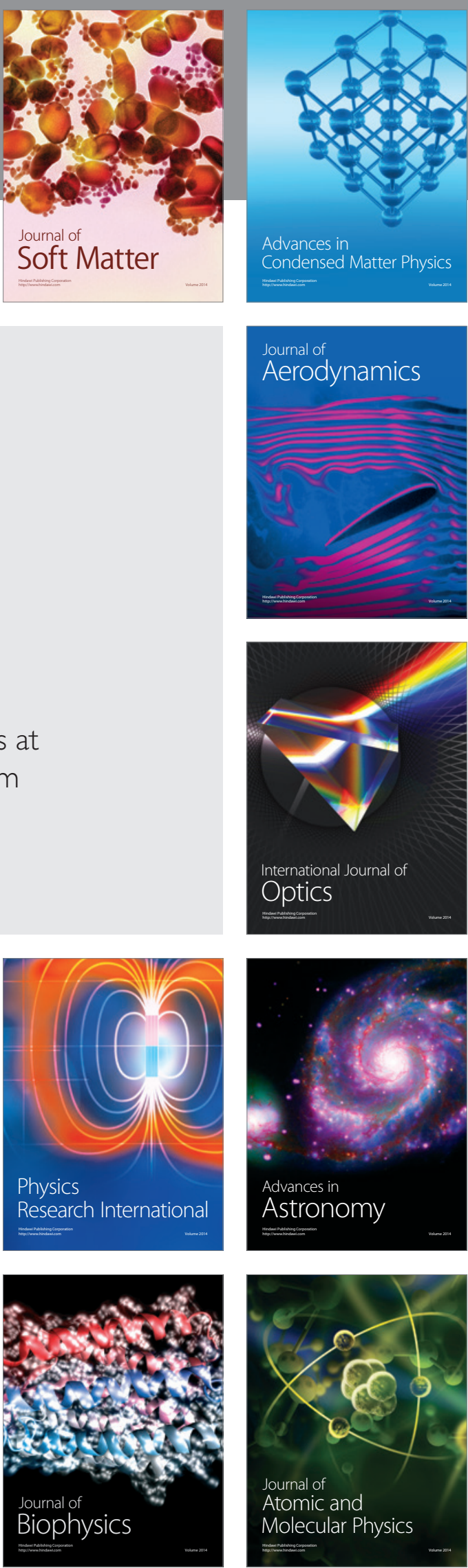